\title{
The Fakeability of Personality Measurement with Graded Paired Comparisons
}

\author{
Niklas Schulte ${ }^{1}$, Lucas Kaup ${ }^{1}$, Paul-Christian Bürkner ${ }^{2}$, and \& Heinz \\ Holling ${ }^{1}$ \\ ${ }^{1}$ University of Münster \\ Germany \\ 2 Aalto University \\ Finland
}

Abstract

This pre-registered study compares the faking resistance of Likert scales and graded paired comparisons (GPCs) analyzed with Thurstonian IRT models. Based on findings on other forced-choice formats, we hypothesized that GPCs would be more resistant to faking than Likert scales by resulting in lower score inflation and better recovery of applicants' true (i.e., honest) trait scores. A total of $N=573$ participants completed either the Likert or GPC version of a personality questionnaire first honestly and then in an applicant scenario. Results show that participants were able to increase their scores in both the Likert and GPC format, though their score inflation was smaller in the GPC than the Likert format. However, GPCs did not exhibit higher honest-faking correlations than Likert scales; under certain conditions, we even observed negative associations. These results challenge mean score inflation as the dominant paradigm for judging the utility of foecedchoice questionnaires in high-stakes situations. Even if FC factor scores are less inflated, their ability to recover true trait standings in high-stakes situations might be lower compared with Likert scales. Moreover, in the GPC format, faking effects correlated almost perfectly with the social desirability differences of the corresponding statements, highlighting the importance of matching statements equal in social desirability when constructing forcedchoice questionnaires.

Keywords: Forced-choice, Thurstonian IRT model, Ipsative data, Graded paired comparisons, Graded-preference items, Compositional items IRT 
This preprint represents an unreviewed version of a manuscript to be formally published in the future. Please direct any comments on possible errors to niklas.schulte@uni-ulm.de.

Niklas Schulte, Department of Psychology, University of Münster, Germany; Lucas Kaup, Department of Psychology, University of Münster, Germany; Heinz Holling, Department of Psychology, University of Münster, Germany; Paul-Christian Bürkner, Department of Computer Science, Aalto University, Finland.

Niklas Schulte is now at Department of Psychology and Education, University of Ulm, Germany.

Acknowledgements. This manuscript is based on the master thesis of Lucas Kaup and is part of the dissertation of Niklas Schulte.

To conduct the presented analyses and create this article, we used the programming language $\mathrm{R}$ (Version 4.0.2; R Core Team, 2020) and the R-packages brms (Version 2.13.5; Bürkner, 2017, 2018), corx (Version 1.0.6.1; Conigrave, 2020), dplyr (Version 1.0.1; Wick- ham et al., 2020), ggplot2 (Version 3.3.2; Wickham, 2016), gridExtra (Version 2.3; Auguie, 2017), kableExtra (Version 1.1.0; Zhu, 2019), lavaan (Version 0.6.7; Rosseel, 2012), mirt (Version 1.32.1; Chalmers, 2012), MVN (Version 5.8; Korkmaz et al., 2014), papaja (Version 0.1.0.9997; Aust \& Barth, 2020), rstan (Version 2.21.2; Stan Development Team, 2020), thurstonianIRT (Version 0.11.1; Bürkner, 2020), tibble (Version 3.0.3; Müller \& Wickham, 2020), tidyr (Version 1.1.1; Wickham \& Henry, 2020), tidyverse (Version 1.3.0; Wickham et al., 2019).

Correspondence concerning this article should be addressed to Niklas Schulte, Department of Psychology and Education, University of Ulm, 89069 Ulm, Germany. E-mail: n.schulte@uni-ulm.de 
Questionnaires designed to measure personality, attitudes, interests, or values usually use self-report measures with Likert scales. On a Likert scale, test-takers have to rate their agreement to self-describing statements, i.e., from 1 (strongly agree) to 5 (strongly disagree; Likert, 1932). However, these items are subject to a number of biases, such as faking in a socially desirable direction, acquiescence (confirmation tendency), exaggerated coherence between items of different traits ("halo" effect), and several others (Paulhus \& Vazire, 2007; Wetzel et al., 2016; Wetzel \& Greiff, 2018).

These distortions may compromise the validity of the conclusions derived from Likert scales (Christiansen et al., 2005), especially in high-stakes situations, such as personnel selection (Birkeland et al., 2006; Christiansen et al., 2005) and performance appraisal (Brown et al., 2017), clinically relevant constructs (Young, 2018), or when the measured traits are particularly undesirable, like in "dark" personality traits (Guenole et al., 2018; Paulhus \& Jones, 2014). Response biases can also be problematic when differentiation is particularly important, such as in market research or career advice (Wang et al., 2017). Problems can also arise in applications like comparative cultural research, where the groups under comparison may differ in these biases (Lee et al., 2002).

As a solution to these issues, forced-choice (FC) response formats have been suggested, in which respondents have to decide between two or more items, thus preventing many Likert scale biases by design (e.g., Hontangas et al., 2015; Jackson et al., 2000; Saville \& Willson, 1991; Wetzel et al., 2016). Forced-choice items, however, are associated with two central problems: ipsativity and loss of information in comparison with Likert scales. A person parameter estimate is ipsative if all measured dimensions add up to the same total for each individual (Clemans, 1966). Therefore, a respondent's score on one dimension depends on his or her score on all other dimensions, making inter-individual comparisons based on ipsative scores questionable (Cattell, 1944; Hicks, 1970). This is highly problematic for many applications of forced-choice questionnaires, e.g., in personnel selection, where the comparison between applicants is the central objective (Johnson et al., 1988).

To derive normative parameter estimates from forced-choice responses, Thurstonian IRT models were introduced (Brown \& Maydeu-Olivares, 2011). An estimate is normative if it indicates the relative trait level compared to other subjects of the population distribution (Cattell, 1944). Nevertheless, the loss of information relative to Likert items remains problematic. Simulation studies suggest that even when scored with Thurstonian IRT models, conventional forced-choice items will yield low reliabilities in most applied conditions (Bürkner et al., 2019; Schulte et al., 2020).

A promising solution that might combine the high information generated by Likert items with the faking resistance of the forced choice format are graded paired comparisons (GPCs). In a GPC, two items are placed on both ends of a rating scale and respondents have to indicate the degree to which they prefer one item over the other. Thus, they are a combination of Likert type rating items and conventional dichotomous forced-choice tasks with two items. The two included items maintain the characteristic property of forced-choice scales in that not all items can be fully endorsed at the same time, because one item's gain is the alternative item's loss. At the same time, the graded response provides information about the relative preference of one item over another, increasing the information about the 
latent traits. To distinguish between FC format types, we use the term discrete FC format to refer to the classical one that can be transferred into binary comparisons (but can consist of more than two items) and the term GPC for two items with a rating scale in between. Figure 1 shows a GPC sample item.

$\begin{array}{lcccccc} & \begin{array}{c}\text { Much } \\ \text { more }\end{array} & \begin{array}{c}\text { Slightly } \\ \text { more }\end{array} & \begin{array}{c}\text { About } \\ \text { the same }\end{array} & \begin{array}{c}\text { Slightly } \\ \text { more }\end{array} & \begin{array}{c}\text { Much } \\ \text { more }\end{array} & \\ \begin{array}{l}\text { I work hard } \\ \text { for my goals. }\end{array} & \bigcirc & \bigcirc & \bigcirc & \bigcirc & \bigcirc & \begin{array}{l}\text { I stay calm } \\ \text { under pressure. }\end{array}\end{array}$

\section{Figure 1}

Example of a graded paired comparison.

For a long time, GPCs were used reluctantly - as were all forced-choice related formats - due to the ipsativity of the scores derived from them; now though, the generalization of the Thurstonian IRT model for graded comparisons (Brown \& Maydeu-Olivares, 2018) was developed to provide non-ipsative parameters for graded preference formats like GPCs. However, little is known about GPCs as a response format for personality measures. Further, although researchers often assume that GPCs offer improved resistance to faking compared with Linkert scales, this is untested, and little knowledge has been gained regarding the application of the Thurstonian IRT model as a scoring method.

Therefore, this study was designed to investigate the potential merits of GPCs over Likert items regarding their ability to resist faking attempts in the context of personnel selection. Additionally, it investigates whether the expected reliability gains are sufficient to achieve a measurement error small enough for individual diagnostic purposes in practice. Although a recent meta-analysis emphasizes the influence of item desirability on the fakeability of forced-choice questionnaires (Cao \& Drasgow, 2019), little is known about what to consider when trying to match items on desirability during the construction of forced-choice item blocks. This may be one of the reasons why desirability matching is often ignored in questionnaire development, giving rise to their fakeability (Bürkner et al., 2019). To inform test constructors about desirability matching, as it is perhaps the most important feature of forced-choice tests, we will investigate in detail the effects of item and trait desirability as well as the association with the keyed direction of items (i.e., the sign of the factor loading). Researchers have argued that when negatively and positively keyed items are combined, one item will typically represent the desired end of the trait continuum and the other item will represent the undesired end (Bürkner et al., 2019; Schulte et al., 2020). Thus, even though simulations suggest that such comparisons of differently keyed items are crucial for sufficient reliabilities in FC-type questionnaires (Brown \& Maydeu-Olivares, 2011; Bürkner et al., 2019; Schulte et al., 2020), they might be easily faked. GPCs are particularly suitable to test this theoretical assumption, because the graduated format even allows to detect nuances of changing preferences due to different social desirabilities of items. 


\section{Graded Paired Comparisons as a Psychometric Method}

In the past, GPCs have primarily been used to investigate consumer preferences (e.g., De Beuckelaer et al., 2013; Agresti, 1992; Alfaro-Rodriguez et al., 2005; Ofir, 2004). For example, in marketing research GPCs are often employed in the context of conjoint analyses (e.g., Scholz et al., 2010). GPCs are also known by slightly different names, such as constant sum paired comparisons (Skedgel et al., 2015) or ordinal paired comparisons (Agresti, 1992).

Recently, Brown and Maydeu-Olivares (2018) proposed applying GPCs in personality assessments, as GPCs entail advantages over discrete forced-choice formats: First, GPCs can lead to greater acceptance by participants, as participants are not forced as harshly to choose between options that might describe them equally (in)appropriately (see Dalal et al., 2019 for more positive applicant reactions). Second, the aforementioned information gain achieved by re-introducing a rating scale might help to increase reliability. Indeed, first applications of the Thurstonian IRT model for graded comparisons found reliability gains between .07 and .10 when switching from discrete forced-choice to GPCs (Brown \& Maydeu-Olivares, 2018). Unfortunately, re-implementing a Likert scale also brings back typical method-related response biases such as extreme responding, but empirical results suggest these effects to be negligible (De Beuckelaer et al., 2013). Further, test-takers still have to contrast the two opposing statements within one comparison, which means they cannot fully endorse all desirable statements. In the following, we will present current findings on the fakeability of questionnaires and discuss what can be expected for GPCs in this respect.

\section{Overall Faking Effects}

Are people able to deliberately distort their answers in personality questionnaires, for instance, in order to present themselves more favorably when applying for a job? The simple answer to this question is yes. According to meta-analyses, respondents can improve their scores substantially if they are instructed to do so (meta-analytically by $d=.73$ in Edens \& Arthur, 2000, and, depending on the trait the effect size ranges between $d=.47$ for agreeableness and $d=.93$ for emotional stability in Viswesvaran \& Ones, 1999). In situations where participants were faced with real-life incentives to distort (e.g., money or a job), the distortion seems to be somewhat weaker $(d=.30$ in a meta-analysis by Edens $\&$ Arthur, 2000). This was also shown in another meta-analysis when faking by job applicants was examined trait-wise $(d=.11$ for extraversion, $d=.44$ for emotional stability, $d=.45$ for conscientiousness and $d=.13$ for openness; Birkeland et al., 2006).

To our knowledge, there is no empirical evidence regarding the specific fakeability of GPCs in personality assessment. However, since the theoretical rationale for GPCs' potential to resist faking is the same as for discrete forced-choice formats, here we review the results of the corresponding research. Concerning the effects of faking on FC measures, a recent meta-analysis yielded an overall effect of $d=.06$ for mean score inflation from honest to faking conditions, which is clearly below the effects reported for Likert scales (Cao \& Drasgow, 2019). This meta-analysis also demonstrates that the effects are smaller 
if jointly presented items are matched for social desirability. Thus, the general mechanism of FC measures intended to reduce faking seems to work. In addition to meta-analyses, which consider the fakeability of one response format (Likert vs. FC) in isolation, individual studies that have drawn a direct comparison can also be used to assess the relative susceptibility to faking. In fact, the vast majority of these studies found faking-induced score inflation to be smaller for FC scales (Christiansen et al., 2005; Huber, 2017; Jackson et al., 2000; Lee et al., 2019; Pavlov et al., 2019; Vasilopoulos et al., 2006); but, see Heggestad et al. (2006) for contrary results.

Taken together, test-takers can inflate their scores in both Likert and FC questionnaires. Because essentially the same mechanism is supposed to inhibit response distortion in FC items and GPCs, we assume that a certain amount of score inflation will also be found in GPCs:

Hypothesis 1: Both rating scales and graded paired comparisons are fakeable. The average trait scores are higher (in the socially desired direction) when respondents try to distort them than when they answer honestly.

Although test-takers can elevate their mean trait scores in FC questionnaires when motivated to do so, the magnitude is significantly smaller than that which can be obtained when using Likert scales. As GPCs adopt the crucial FC characteristic of contrasting different statements, they should also make it harder to endorse all desirable items, thus impeding faking. We therefore expect:

Hypothesis 2: Graded paired comparisons exhibit a higher resistance to faking attempts than Likert scales.

When analyzing faking effects on questionnaires, two different approaches can be taken: The first is mean score inflation, as described in the previous section; this is calculated by subtracting the honest score from the faked score and then dividing the result by the standard deviation of the honest scores. The second approach is to assess the correlation between honest and faked trait scores (Pavlov et al., 2018). We will test fakeability with both approaches. As we have already discussed, we expect lower mean score inflation in the GPC condition.

Hypothesis 2a: The mean score inflation between honest and faked answers is lower in graded paired comparisons than in Likert scales.

The correlation between scores of the honest and faking condition is a second measure in which a potentially enhanced resistance to faking attempts should be manifested. It is particularly important for potential faking in personnel selection, since faking in this context would be closely linked to changes in applicants' rank order and, therefore, is directly relevant to selection decisions. While one study, showed that FC scores from an applicant condition explained more "true" trait variance than their Likert scale counterparts (Christiansen et al., 2005), other studies have failed to consistently show that discrete FC scales 
are superior (Guan, 2015; Heggestad et al., 2006; Pavlov et al., 2019). This might be a consequence of reliability issues associated with discrete FC measures (Guan, 2015; Pavlov et al., 2019) or for the study by Heggestad and colleagues (2006), an artifact of ipsative scoring methods. Importantly, reliability issues could be counteracted by the GPC format, and ipsative scoring artifacts could be avoided by scoring responses with Thurstonian IRT models as this would facilitate normative trait score estimates. Furthermore, in the study by Heggestad et al. (2006), honest scores were obtained exclusively with a Likert scale, resulting in a common method bias in favor of Likert scales. Thus, we assume that GPCs can preserve more of the test-takers "true" variance than can Likert scales when using a study design that obtains data in both honest and faking conditions for both Likert and GPC items, whereby the GPC are analyzed with Thurstonian IRT models yielding normative scores:

Hypothesis 2b: The correlation of honest and faked trait scores will be higher in graded paired comparisons compared with Likert scales.

\section{The Role of Individual Faking Tendency}

Identifying mean score inflation is a group-level approach, in that it describes how much scores change on average. However, not every respondent intends to fake their answers: In personnel selection contexts, estimates suggest that about $40 \%$ to $60 \%$ of applicants actually alter their scores (Donovan et al., 2003; Griffin \& Wilson, 2012; Griffith et al., 2007). Further, there is considerable variance in the extent to which respondents fake their answers (Rosse et al., 1998). Even in studies where participants are asked to fake as much as possible, test takers differ in the degree to which they fake (e.g., McFarland \& Ryan, 2000; Pavlov, 2015). As individual faking tendency increases, the relationship between honest and faked scores decreases in discrete FC formats (Pavlov, 2015; Pavlov et al., 2019). We assume this to be the case for GPCs, too:

Hypothesis 3: As the individual faking tendency increases, the association of honest and faked scores will decrease across the two questionnaire formats.

While the theory on response distortion and the empirical findings for discrete FC tasks are in agreement here, another observation calls for more research: In the Pavlov et al. (2019) study, the association of honest and faked trait scores decreased with an increasing individual faking tendency for both the Likert and the FC formats to an equal extent. To explain this, the authors mention the lower reliability of FC measurements or the fact that manipulations of one trait indicator in FC inevitably lead to changes in another trait's indicator, which could not be distinguished in their design (Pavlov et al., 2019). The increased reliability of GPCs (Brown \& Maydeu-Olivares, 2018) might allow one to test whether the empirical results are in line with the previous theory on the fakeability of FC formats when the reliabilities of both formats are comparable. In line with the assumption that FC techniques can resist even stronger faking attempts we hypothesize: 
Hypothesis 4: The association of honest and faked trait scores will be less affected by the individual faking tendency in the GPC format compared with the Likert format.

\section{Social Desirability of Items}

Finally, we will investigate possible effects of the perceived social desirability of the statements on their fakeability. We assume that forcing respondents to choose between equally desirable items is the mechanism that helps prevent faking in $\mathrm{FC}$ questionnaires. However, many scholars do not match their items for social desirability at all (Cao \& Drasgow, 2019). This tendency is amplified by the requirements of Thurstonian IRT models, whose reliability critically depends on the inclusion of unequally keyed items in the questionnaires (Brown \& Maydeu-Olivares, 2011; Bürkner et al., 2019; Schulte et al., 2020). Unequally keyed item blocks contain positively and negatively keyed items that are unlikely equally desirable. We believe that this basic assumption about the functioning of FC, which has so far hardly been empirically tested, should be examined more closely, as it has fundamental implications for the test design and the usefulness of the Thurstonian IRT approach. The impact of desirability differences on faking behavior can be tested much more efficiently with GPCs than with discrete FC formats, because responses shift on a much more fine-grained ordinal scale, such that they can even detect effects of small desirability differences. Thus, we will test the following hypothesis:

Hypothesis 5: The mean score inflation for a given graded paired comparison is positively correlated with the difference in perceived desirability of its two statements.

The hypotheses, the method and the data analysis procedure of this study were specified prior to the start of data collection. For the preregistration form, see https: //osf.io/8emj7.

\section{Method}

\section{Participants}

Based on a power analysis, the intended sample size was 618 observations, giving an expected effect size of $d=.16$ for the mean score inflation in FC questionnaires (see meta-analysis by Cao \& Drasgow, 2019), an $\alpha$ error of .05, a power of .80, and equal sample sizes in all conditions. Participants were recruited via an online panel of professionals (Psy$W e b$ ), social media channels (Facebook, Xing, LinkedIn, Instagram), the online platform SurveyCircle, and the student participant pools at the universities of Münster and Ulm. The data collection took place between September 2019 and February 2020.

Of the original 591 participants, seven were excluded because they were younger than 18 years, one person's German skills were insufficient, and five individuals wanted to exclude their data from the analysis after they had completed the study. Moreover, three 
people were excluded because their response time was less than one third of the average in the GPC condition and the $S D$ of their responses was more than three $S D s$ below the average $S D$. Furthermore, one person was excluded in the GPC condition solely due to a very short overall response time. In the Likert condition, one person was excluded because their responses' $S D$ was more than three $S D s$ below the average $S D$ and their response time was only $41 \%$ of the average response time in their condition.

Thus, the final sample size was $N=573$ ( $78 \%$ female). Participants' ages ranged from 18 to $56(M=27.50, S D=9.93)$. In our sample, the majority $(67 \%)$ were students, $20 \%$ were employed full-time and $9 \%$ were part-time employees, while $4 \%$ were unemployed. The highest educational level was a secondary school certificate for $3 \%$, a university entrance qualification for $61 \%$, and a university degree for $36 \%$ of the participants. As an incentive, participants were offered feedback on their ability to fake in applicant settings and psychology students could earn course credits. Additionally, the four "best" applicants were rewarded with $50 €$ each.

\section{Design}

We conducted an online experiment with a 2x2 mixed design. Participants completed a GPC personality measure $(n=283)$ or the Likert version of the same questionnaire $(n=290)$. We used a between-subject design for this factor to ensure sufficient attention of the participants over the entire duration of the online experiment. After the participants had been asked to answer honestly, we presented a job advertisement for the position of a project manager. The participants were then asked to answer the same questionnaire again, but in a way that maximized their chances of getting the job. We used a withinsubject design for the honest/faking factor, as several of our hypotheses had to be tested with regression analysis. Additionally, meta-analyses show that this design can detect faking effects better (Edens \& Arthur, 2000; Viswesvaran \& Ones, 1999). Respondents were randomly assigned to the two response format conditions, with equal group sizes being enforced with the last participants. In the end of the applicant condition, respondents were asked how much they tried to fake their answers (referred to as individual faking tendency in the following). Finally, both groups rated the desirability of the traits that had been measured and the personality items that had been used on a rating scale. Participants from the Likert condition additionally rated the relative desirability of the two items for 28 pairs of items that formed a GPC. This was done to identify possible context effects on social desirability evoked by directly comparing an item with another item. To adjust the work-load of the two conditions, these questions were presented exclusively in the Likert group, as the GPC version of the personality questionnaire was considerably more exhausting and time consuming than the Likert version $\left(M_{G P C}=44\right.$ min vs. $\left.M_{\text {Likert }}=24 \mathrm{~min}\right)$. For the wording of the instructions, the order of the items, etc., see the code book on OSF (https://osf.io/fx4yz/?view_only=6fa25e11aab64d5080c3ec4a1bbf6019). 
Table 1

Reliabilities for Likert Items and Graded Paired Comparisons

\begin{tabular}{|c|c|c|c|c|c|c|}
\hline & \multicolumn{3}{|c|}{ Reliability } & \multicolumn{3}{|c|}{$R M S E$} \\
\hline & Overall & Honest & Faking & Overall & Honest & Faking \\
\hline \multicolumn{7}{|l|}{ Likert } \\
\hline Emotional Stability & 0.96 & 0.92 & 0.91 & 0.20 & 0.30 & 0.40 \\
\hline Extraversion & 0.95 & 0.93 & 0.87 & 0.24 & 0.28 & 0.37 \\
\hline Agreeableness & 0.88 & 0.85 & 0.87 & 0.35 & 0.40 & 0.45 \\
\hline Conscientiousness & 0.95 & 0.92 & 0.83 & 0.25 & 0.31 & 0.61 \\
\hline \multicolumn{7}{|c|}{ GPC (unequ. and equ. keyed) } \\
\hline Emotional Stability & 0.97 & 0.94 & 0.93 & 0.17 & 0.24 & 0.27 \\
\hline Extraversion & 0.97 & 0.96 & 0.93 & 0.19 & 0.21 & 0.23 \\
\hline Agreeableness & 0.93 & 0.90 & 0.93 & 0.26 & 0.32 & 0.34 \\
\hline Conscientiousness & 0.97 & 0.95 & 0.94 & 0.17 & 0.23 & 0.25 \\
\hline \multicolumn{7}{|l|}{ GPC (equally keyed) } \\
\hline Emotional Stability & 0.89 & 0.79 & 0.84 & 0.37 & 0.54 & 0.54 \\
\hline Extraversion & 0.87 & 0.77 & 0.83 & 0.40 & 0.48 & 0.48 \\
\hline Agreeableness & 0.84 & 0.74 & 0.83 & 0.44 & 0.56 & 0.56 \\
\hline Conscientiousness & 0.91 & 0.84 & 0.88 & 0.33 & 0.48 & 0.48 \\
\hline
\end{tabular}

Note. RMSE = root mean squared individual measurement error; GPC = graded paired comparisons; unequ. and equ. keyed = questionnaire with both unequally and equally keyed item pairs. Reliabilities for both formats were calculated with the empirical reliability function from the $\mathrm{R}$ package mirt (Chalmers, 2012). They represent the proportion of estimated true variance on the sum of the estimates for the true variance and the squared mean standard error. The overall estimates for reliability and RMSEs are based on honest and faked scores of all participants, which means that each person is included twice in these calculations. The RMSEs in the honest and faking condition are scaled with the same SD as the corresponding factor scores and can be interpreted on their scale.

\section{Materials}

We used work-related personality items developed for a personnel selection test in a large German governmental organization. They measured the four dimensions of the Big 5 that predict work performance for the main job profile in this organization, namely emotional stability, extraversion, agreeableness, and conscientiousness.

\section{Likert personality questionnaire}

The questionnaire in the Likert condition consisted of 42 self-describing statements, 10 each measuring extraversion and agreeableness and 11 each measuring neuroticism and conscientiousness. Participants indicated their level of agreement on a seven-point Likert 
scale from 1 (do not agree at all) to 7 (fully agree). Empirical reliabilities are reported in Table 1 and can be interpreted as high. Comparisons of measurement accuracy between conditions should be made on the basis of scale-independent reliability estimates and not on the basis of SEs, as the variance of factor scores differs between conditions (see Table 5 in the online supplement).

\section{GPC personality questionnaire}

The questionnaire in the GPC condition consisted of the same items as the Likert version combined in 119 item pairs (95 two-dimensional, 24 unidimensional) of which 48 (two-dimensional) GPCs were unequally keyed. The main analysis and hypotheses tests are all based on this long questionnaire with both equally and unequally (i.e., mixed) keyed items. However, for additional explorative analysis (see results section), the unequally keyed items were excluded, resulting in a questionnaire of 71 equally keyed item pairs. Participants were asked to express the degree to which they prefer one of the two statements within a GPC over the other on a nine-point rating scale ranging from 1 (the statement on the left describes me very much better) to 9 (the statement on the right describes me very much better $)$. The mean standardized factor loading ${ }^{1}$ was $M=0.72(S D=0.12)$ for the mixed keyed questionnaire (containing both equally and unequally keyed item pairs), which is a similar level compared with the CFA-based loadings of Likert items of $M=0.73$ $(S D=0.17)$. Factor loadings for the equally keyed GPC questionnaire were $M=0.84$ $(S D=0.06)$. Indicators of measurement precision for both the mixed keyed and equally keyed questionnaire are reported in Table 1. The GPC reliabilities were on the same level or slightly higher and the SEs were equal or lower than those for Likert scales. However, this comparison should be interpreted with caution, because GPC scales were longer and included more items than Likert scales in our study. Nevertheless, it can be stated that the GPCs achieved excellent reliability, especially when both equally and unequally keyed items were included.

\section{Job advertisement}

A job advertisement for the position of a project manager was used to create a realistic context for the faking condition that is similarly appealing for persons with varying professional backgrounds. It consisted of a general task description and a desired personality/skill profile. The profile listed four general competencies, each corresponding to one of the measured dimensions (e.g., for agreeableness: You are cooperative and sensitive to the needs of your team members). The job description was designed in such a way that the participants would perceive all measured characteristics as equally desirable for the job described.

\footnotetext{
${ }^{1}$ Factor loadings of GPCs were standardized by $\lambda_{\text {standardized }}=\frac{\lambda_{\text {unstandardized }}}{\sqrt{\lambda_{\text {unstandardized }}^{2}+\psi^{2}}}$, where $\lambda$ is the factor loading and $\psi$ is the item uniqueness (i.e., the error). Means are based on absolute values of factor loadings.
} 


\section{Individual faking tendency}

As proposed by Pavlov (2015), the individual tendency to fake was measured by a single item: Participants were asked to what extent they faked their answers in order to present themselves positively with respect to the position of a project manager. The item used an 11-point rating scale ranging from 0 ( $I$ did not distort my answers at all) to 10 ( $I$ distorted my answers strongly). In all regression analyses, faking tendency was $z$-standardized across both formats.

\section{Perceived social desirability}

To obtain a comprehensive understanding of the different facets of the perceived social desirability, we conducted a threefold assessment where, in each of the three steps, participants were instructed to rate the desirability with respect to the position of a project manager. First, participants rated the desirability of each of the 42 single items on a sevenpoint scale ranging from 1 (very undesirable) to 7 (very desirable). Second, they rated the desirability of the four global traits measured in the questionnaire on the same rating scale as the single items. Third, the relative desirability of items in 24 different-trait pairs and 4 same-trait pairs was assessed in graded paired comparisons. These 28 equally keyed pairs were the first items of the original GPC questionnaire that formed an item set balanced for trait frequency. Participants were asked to respond on a nine-point rating scale ranging from 1 (the left statement is much more desirable for the position of a project manager) to 9 (the right statement is much more desirable for the position of a project manager). This method yields a measure for the item desirability within the context of a GPC, as the desirability of a statement may change depending on the statement it is compared with (Waters, 1965).

\section{Trait Score Estimation and Standardization}

In this section, we briefly describe the estimation procedures for participants' trait scores in the Likert and GPC conditions as well as the standardization procedures, which are essential for interpreting the results.

\section{Trait Score Estimation}

We estimated GPC trait scores (person parameters) with Thurstonian IRT models for graded preference data (Brown \& Maydeu-Olivares, 2011, 2018) with the thurstonianIRT package (Bürkner, 2020) in R (R Core Team, 2020). Within this package, we specified an ordinal model using the Bayesian software Stan (Carpenter et al., 2017) as the underlying engine and the EAP estimator. We estimated honest and faking factor scores in a common model to estimate both types of scores on the same scale. Only to test whether the assumption of identical item parameters in both conditions affects the results notably, we estimated one model for each of the two conditions (Zhang et al., 2020), resulting in highly correlated factor loadings in both models $(r=.98)$. All further analyses are based on the joint model. Two participants reached extreme values on several traits and were excluded 
from factor score-based regression analyses (Hypotheses 1 to 4) without consequences for acceptance or rejection of hypotheses.

To keep GPC and Likert scores as comparable as possible, trait scores for the Likert condition were estimated using a multidimensional graded response IRT model (Samejima, 1969), which is ordinal as well. As software we used the R package mirt (Chalmers, 2012) with the Metropolis-Hastings Robbins-Monro (MHRM) algorithm and MAP factor scores. Sensitivity analyses showed no differences between the selected MHRM estimator and other methods for multidimensional models, namely Monte Carlo Expectation Maximization and Quasi Monte Carlo Expectation Maximization. The estimates of all methods correlated to $r=.99$ or higher.

\section{Trait Score Standardization}

For the sake of interpretability, we standardized all trait scores with the mean and standard deviation of the honest scores (within each questionnaire format). As a consequence, each trait score can be interpreted as the difference from the average honest score in honest $S D$ s. For example, if person A has a faking (i.e., applicant) score of 2 on extraversion, this means that this score is two honest $S D$ s above the average honest score on extraversion. Likewise, Likert and GPC honest scores will have a mean of zero and a standard deviation of one, while the mean of the faked scores will reflect the average difference of Likert/GPC faked scores from the average Likert/GPC honest score in honest $S D$ s. Therefore, the means of the faked scores can be interpreted as Cohen's $d$ effect sizes. The data, a code book, and the $\mathrm{R}$ code for all analyses are available on OSF (https://osf.io/fx4yz/?view_only=6fa25e11aab64d5080c3ec4a1bbf6019).

\section{Results}

As intended by the experimental manipulation (job profile), all traits were perceived as highly desirable for the position in the job advertisement $(M=6.61, S D=0.84$ for emotional stability, $M=6.18, S D=0.95$ for extraversion, $M=5.73, S D=1.17$ for agreeableness, and $M=6.82, S D=0.59$ for conscientiousness; all measured on a sevenpoint scale). Agreeableness had comparatively lower but still high trait desirability ratings. The faking instruction within the applicant scenario combined with the financial incentive also appears to have worked successfully: The individual faking tendency was relatively high, with an average value of $7.71(S D=2.30)$ in the Likert and $7.73(S D=2.53)$ in the GPC condition, both measured on a scale from 1 to 11 . The faking tendency did not significantly differ between groups $(\Delta M=0.02,95 \%$ CI $[-0.37,0.42], t(569.23)=0.10, p=.920)$. Table 2 shows the inter-correlations of trait estimates for GPCs (lower triangle) and Likert scales (upper triangle) for the honest and faking condition. GPC inter-trait correlation estimates are similar but not equal when compared with their Likert counterparts. Intertrait correlations are higher in the faking condition than in the honest condition, which is

a typical effect in application situations and indicates that an ideal applicant factor has formed in both formats. 
Table 2

Correlation Matrix for GPC (Lower Triangle) and Likert (Upper Triangle) Factor Scores

\begin{tabular}{lllllllll}
\hline & \multicolumn{1}{c}{1} & \multicolumn{1}{c}{2} & \multicolumn{1}{c}{3} & \multicolumn{1}{c}{4} & \multicolumn{1}{c}{5} & \multicolumn{1}{c}{6} & \multicolumn{1}{c}{7} & \multicolumn{1}{c}{8} \\
\hline 1. Es (honest) & - & $.27^{* * *}$ & .10 & $.38^{* * *}$ & $.16^{* *}$ & .02 & .02 & .05 \\
2. Ex (honest) & $.59^{* * *}$ & - & $.17^{* *}$ & $.34^{* * *}$ & .06 & $.23^{* * *}$ & $.17^{* *}$ & .11 \\
3. Ag (honest) & $.20^{* * *}$ & $.15^{*}$ & - & $.16^{* *}$ & $.14^{*}$ & $.19^{* *}$ & $.30^{* * *}$ & $.16^{* *}$ \\
4. Co (honest) & $.39^{* * *}$ & $.25^{* * *}$ & $.30^{* * *}$ & - & $.17^{* *}$ & .06 & $.13^{*}$ & $.19^{* *}$ \\
5. Es (fake) & .04 & .10 & $.28^{* * *}$ & $.19^{* *}$ & - & $.73^{* * *}$ & $.48^{* * *}$ & $.86^{* * *}$ \\
6. Ex (fake) & -.06 & .09 & $.28^{* * *}$ & $.15^{*}$ & $.82^{* * *}$ & - & $.51^{* * *}$ & $.78^{* * *}$ \\
7. Ag (fake) & .04 & $.15^{*}$ & $.39^{* * *}$ & $.22^{* * *}$ & $.64^{* * *}$ & $.59^{* * *}$ & - & $.55^{* * *}$ \\
8. Co (fake) & .02 & .09 & $.29^{* * *}$ & $.18^{* *}$ & $.82^{* * *}$ & $.74^{* * *}$ & $.65^{* * *}$ & - \\
\hline
\end{tabular}

Note. Es = emotional stability; Ex = extraversion; $\mathrm{Ag}=$ agreeableness; $\mathrm{Co}=$ conscientiousness; honest $=$ honest condition; fake $=$ faking condition. Upper triangle shows Likert and lower triangle shows GPC factor score correlations. ${ }^{*} \mathrm{p}<0.05 ;{ }^{*} \mathrm{p}<0.01$; $* * * \mathrm{p}<0.001$

Due to the inter-correlations of traits (Table 2) and the heterogeneity of variances, we tested all hypotheses with multivariate linear models that account for heterogeneous error variances across the range of predictors. We estimated all multivariate models with the $\mathrm{R}$ package brms for Bayesian regression modeling (Bürkner, 2017, 2018) based on Stan (Carpenter et al., 2017). See the online supplement on https://osf.io/fx4yz/?view_only= 6fa25e11aab64d5080c3ec4a1bbf6019 for model equations. Aside from modeling heterogeneous variances, the multivariate analyses also allow for estimating residual covariances between the dependent variables (i.e., the four traits). The regression coefficients of these models can be interpreted like those of single multiple regressions, but credible intervals take into account the inter-correlations of traits. In the Bayesian analyses, we consider as significant those results where the value of the null hypothesis is outside the $95 \%$ credible interval. Since the methods mentioned above model the data better than the pre-registered tests for Hypotheses 1 and 2 ( $t$ tests and bivariate correlations; https://osf.io/fx4yz/?view only $=6 \mathrm{fa} 25 \mathrm{e} 11 \mathrm{aab} 64 \mathrm{~d} 5080 \mathrm{c} 3 \mathrm{ec} 4 \mathrm{a} 1 \mathrm{bbf6019})$ but at the same time maintain the basic idea of the pre-registered methods, we decided to deviate from the original analysis plan in this respect. We report results for the original analysis plan in an online supplement on OSF (https://osf.io/fx4yz/?view_only=6fa25e11aab64d5080c3ec4a1bbf6019). The adjustments to the analysis plan had no effect on the acceptance or rejection of hypotheses. Our analysis approach is based on the regression-based moderation framework suggested by Pavlov et al. (2019).

Hypothesis 1 stated that average trait scores are higher when respondents try to distort them than when they answer honestly. Table 3 shows the model results for the regression of trait scores on the condition (dummy coded with honest $=0$ and faking $=1$ ). The intercepts represent the predicted values for the honest condition, which are zero for all traits due to the standardization procedure. The slope coefficients represent the predicted 


\section{Table 3}

Multivariate Regression Results with the Condition as the Predictors for Trait Scores

\begin{tabular}{lcc}
\hline & Intercept (honest) & Slope (faking) \\
\hline Likert & & \\
Emotional Stability & $0.00[-0.13,0.14]$ & $2.67[2.49,2.85]$ \\
Extraversion & $0.00[-0.13,0.14]$ & $2.00[1.83,2.17]$ \\
Agreeableness & $0.00[-0.13,0.12]$ & $0.97[0.78,1.15]$ \\
Conscientiousness & $0.00[-0.14,0.15]$ & $3.11[2.91,3.30]$ \\
GPC & & \\
Emotional Stability & $-0.02[-0.16,0.13]$ & $2.15[1.97,2.31]$ \\
Extraversion & $-0.02[-0.15,0.12]$ & $1.51[1.35,1.67]$ \\
Agreeableness & $-0.03[-0.15,0.09]$ & $1.16[0.99,1.34]$ \\
Conscientiousness & $-0.03[-0.16,0.11]$ & $2.04[1.88,2.21]$ \\
\hline
\end{tabular}

Note. Faking $=$ Factor score in faking condition; $\mathrm{GPC}=$ Graded paired comparison. Condition is dummy coded with honest $=0$ and faking $=1$. Each column reports regression coefficients followed by the corresponding $95 \%$ credible interval [in brackets]. Results are based on separate models for each questionnaire format (Likert and GPC).

values for the faking condition and can be interpreted as Cohen's $d$ effect sizes. Across all traits, effects for both formats are large and 95\% CIs for slope parameters (i.e., the differences between honest and faking condition) do not include zero. Thus, results support Hypothesis 1; that is, participants were able to fake both questionnaire types when they were instructed to do so.

Hypothesis 2a stated that GPC scores are less inflated than Likert scores. To test this hypothesis, we predicted the faking scores, which can be interpreted as the scores' inflation from the honest to the faking condition, by the questionnaire format (dummy coded with Likert $=0$ and GPC $=1$ ) while controlling for honest scores and the interaction of honest scores and questionnaire format. At an honest score of 0 , the score inflation was significantly lower for GPCs than for Likert scales in three out of four traits (see credible intervals for format coefficients in Table 4), largely supporting Hypothesis 2a.

Hypothesis $2 \mathrm{~b}$ stated that the correlation of honest and faked scores would be higher in GPCs compared with Likert scales. We tested this on the basis of the interaction term for the honest score and the questionnaire format (see Table 4). Although all interaction terms are significantly different from 0 for all traits but agreeableness, their signs indicate an effect contrary to the hypothesis: The association of honest and faked scores is higher for Likert scales than for GPCs. This is consistent with the results for trait-wise direct comparisons of the honest-faking correlations for Likert scales and honest-faking correlations for GPCs (see online supplement on https: 
Table 4

Multivariate Multiple Regression Results with the Interaction of Honest Scores and Format as Predictors for Faked Scores

\begin{tabular}{ccccc}
\hline & Intercept & Honest & Format & Honest*Format \\
\hline Es & $2.67[2.54,2.80]$ & $0.21[0.14,0.29]$ & $-0.56[-0.73,-0.39]$ & $-0.12[-0.22,-0.02]$ \\
Ex & $2.01[1.90,2.11]$ & $0.19[0.13,0.25]$ & $-0.51[-0.65,-0.38]$ & $-0.13[-0.22,-0.04]$ \\
Ag & $0.97[0.83,1.10]$ & $0.28[0.16,0.39]$ & $0.16[-0.03,0.35]$ & $0.01[-0.16,0.18]$ \\
Co & $3.11[2.96,3.25]$ & $0.17[0.09,0.25]$ & $-1.09[-1.28,-0.91]$ & $-0.17[-0.29,-0.07]$ \\
\hline
\end{tabular}

Note. ES = emotional stability; Ex = extraversion; Ag = agreeableness; Co = conscientiousness; Honest $=$ honest scores; Each column reports regression coefficients followed by the corresponding $95 \%$ credible interval [in brackets]. Format is dummy coded with Likert $=0$ and graded paired comparisons $=1$. Honest*Format is the interaction effect of these two variables.

//osf.io/fx4yz/?view_only=6fa25e11aab64d5080c3ec4a1bbf6019). They also do not support Hypothesis $2 \mathrm{~b}$.

\section{The Role of Individual Faking Tendency}

Next, we analyzed the impact of participants' individual faking behavior. We hypothesized that the association of participants' honest and faked scores will decrease the more they distort their responses in the faking condition, regardless of the test format (Hypothesis 3). To investigate this hypothesis, we ran standardized multivariate multiple regression models for Likert and GPC formats. Faked trait scores were regressed on corresponding honest scores, individual faking tendency, and the interaction of these variables. Assuming a positive regression coefficient for honest scores, the interaction term should have a negative sign, thus reducing the regression weight of honest scores as faking tendency increases.

The interaction coefficients were negative in all cases and significant for all traits but agreeableness in the Likert format and for all traits in the GPC format (see CIs for the interaction terms in Table 5). Figure 2 plots the interactions of honest scores and individual faking tendency. For Likert scales (upper row of plots) we see that the slope becomes flatter the higher the faking tendency, that is, the more respondents try to fake, the less the "true" values are mirrored in their faked responses. The same is true for GPCs (lower row), but here we see that when the faking tendency is high (i.e., one SD above the mean), the faked scores are actually lower than the honest scores of the same individual (see negative slopes in three out of four traits). We will return to this point in the explorative results section.

The differences between the Likert and the GPC format concerning the effects of faking tendency were addressed by Hypothesis 4: We hypothesized that the individual faking tendency would affect the association between honest and faked scores in GPC less than in the Likert format. To examine this issue, we again ran multivariate multiple regression 


\section{Table 5}

Multivariate Multiple Regression Results with the Interaction of Honest Scores and Individual Faking Tendency as Predictors for Faked Scores

\begin{tabular}{ccccc}
\hline & Intercept & Honest & Faking tendency & Honest*faking tend. \\
\hline Likert & & & & \\
Es & $2.64[2.51,2.77]$ & $0.21[0.13,0.29]$ & $0.45[0.31,0.59]$ & $-0.13[-0.21,-0.05]$ \\
Ex & $1.98[1.88,2.08]$ & $0.19[0.13,0.25]$ & $0.36[0.26,0.46]$ & $-0.15[-0.20,-0.09]$ \\
Ag & $0.97[0.83,1.10]$ & $0.29[0.17,0.41]$ & $0.15[0.01,0.28]$ & $-0.09[-0.20,0.02]$ \\
Co & $3.07[2.93,3.22]$ & $0.18[0.11,0.26]$ & $0.50[0.34,0.65]$ & $-0.14[-0.21,-0.07]$ \\
GPC & & & & \\
Es & $2.08[1.97,2.18]$ & $0.11[0.05,0.17]$ & $0.30[0.20,0.41]$ & $-0.15[-0.21,-0.10]$ \\
Ex & $1.46[1.37,1.55]$ & $0.08[0.02,0.14]$ & $0.23[0.14,0.31]$ & $-0.17[-0.23,-0.10]$ \\
Ag & $1.10[0.96,1.25]$ & $0.24[0.12,0.36]$ & $0.10[-0.02,0.22]$ & $-0.19[-0.29,-0.08]$ \\
Co & $1.98[1.87,2.09]$ & $0.03[-0.06,0.11]$ & $0.26[0.15,0.36]$ & $-0.18[-0.26,-0.11]$ \\
\hline
\end{tabular}

Note. $\mathrm{ES}=$ emotional stability; $\mathrm{Ex}=$ extraversion; $\mathrm{Ag}=$ agreeableness; $\mathrm{Co}=$ conscientiousness; Honest $=$ honest scores; Honest*faking tend. = interaction of honest score and faking tendency. Each column reports regression coefficients followed by the corresponding $95 \%$ credible interval [in brackets]. Faking tendency is z transformed. Honest*Faking Tendency is the interaction effect of these two variables.

analyses regressing faked trait scores on the format (Likert vs. GPC), the honest scores, individual faking tendency, and all possible interactions of these variables.

The relevant statistics for Hypothesis 4 are the regression coefficients of the threefold interaction Format $\times$ Honest Scores $\times$ Faking Tendency. As shown in the section on Hypothesis 3, the association of honest and faked scores - that is, the regression weight for the predictor Honest Scores - becomes smaller as faking tendency increases (= negative coefficient for Honest Scores $\times$ Faking Tendency interaction term). If this effect is more pronounced in case of the Likert format, the coefficient of the threefold interaction should be of positive magnitude, thus shifting the coefficient of the interaction Honest Scores $\times$ Faking Tendency closer to zero when switching from the Likert format (reference group) to the GPC format. However, the threefold interaction effects were non-significant for all four traits (regression coefficients and 95\% credible intervals were $-0.01[-0.10,0.09]$ for emotional stability, $0.00[-0.08,0.08]$ for extraversion, $-0.05[-0.19,0.09]$ for agreeableness, and $-0.01[-0.11,0.09]$ for conscientiousness). Thus, Hypothesis 4 is not supported. For all coefficients of the regression model, see the online supplement.

\section{The Role of Perceived Item Desirability}

Hypothesis 5 dealt with the response process of the GPC format. We hypothesized that the mean score inflation for a given GPC is positively associated with the difference in perceived desirability of its two statements. To test this, we computed the difference of 

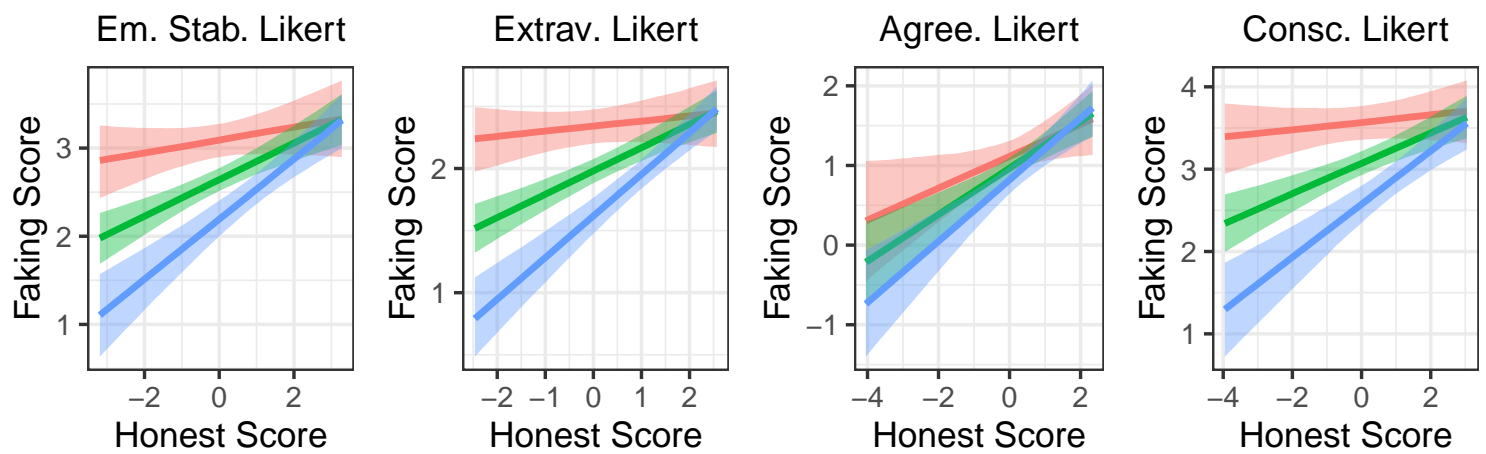

Em. Stab. GPC
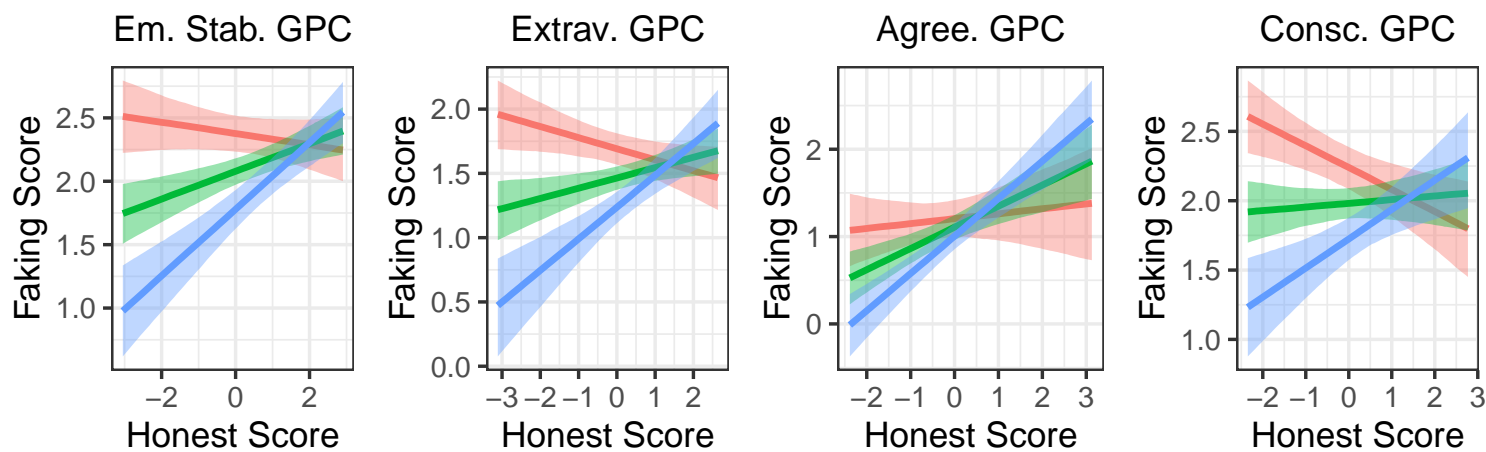

Faking Tendency (z standardized)
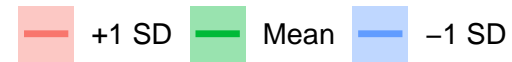

Figure 2

Interaction plots for Hypothesis 3. Em. Stab. = emotional stability; Extrav. = extraversion; Agree. = agreeableness; Consc. = conscientiousness; GPC = graded paired comparison condition. Shaded areas represent $95 \%$ credible intervals.

perceived desirability for each GPC by subtracting the average perceived desirability of the left statement from the average perceived desirability of the right statement within each GPC. We also calculated the mean score inflation for each GPC by subtracting the raw mean score of a given GPC in the honest condition from its corresponding raw mean score in the faking condition. Therefore, the higher the absolute value of the score inflation, the more the mean for this item has changed between the honest and faking condition.

The correlation between desirability difference of both items in a GPC and score inflation in this GPC is $r=.94,95 \%$ confidence interval $[.91, .96], t(117)=29.21, p<$ .001. Moreover, the intercept of the unstandardized regression of the score inflation on the desirability difference is $b=0.10,95 \%$ confidence interval $[0.00,0.21]$. Thus, when both items are equally desirable (i.e., their desirability difference is zero), the predicted score inflation is only .10 SDs. Results are graphically displayed in Figure 3. In this figure, blue points represent equally keyed GPCs and red points represent unequally keyed GPCs. Equally keyed GPCs clearly show less absolute score inflation than unequally keyed GPCs, forming nearly perfectly separable classes with very little overlap. 


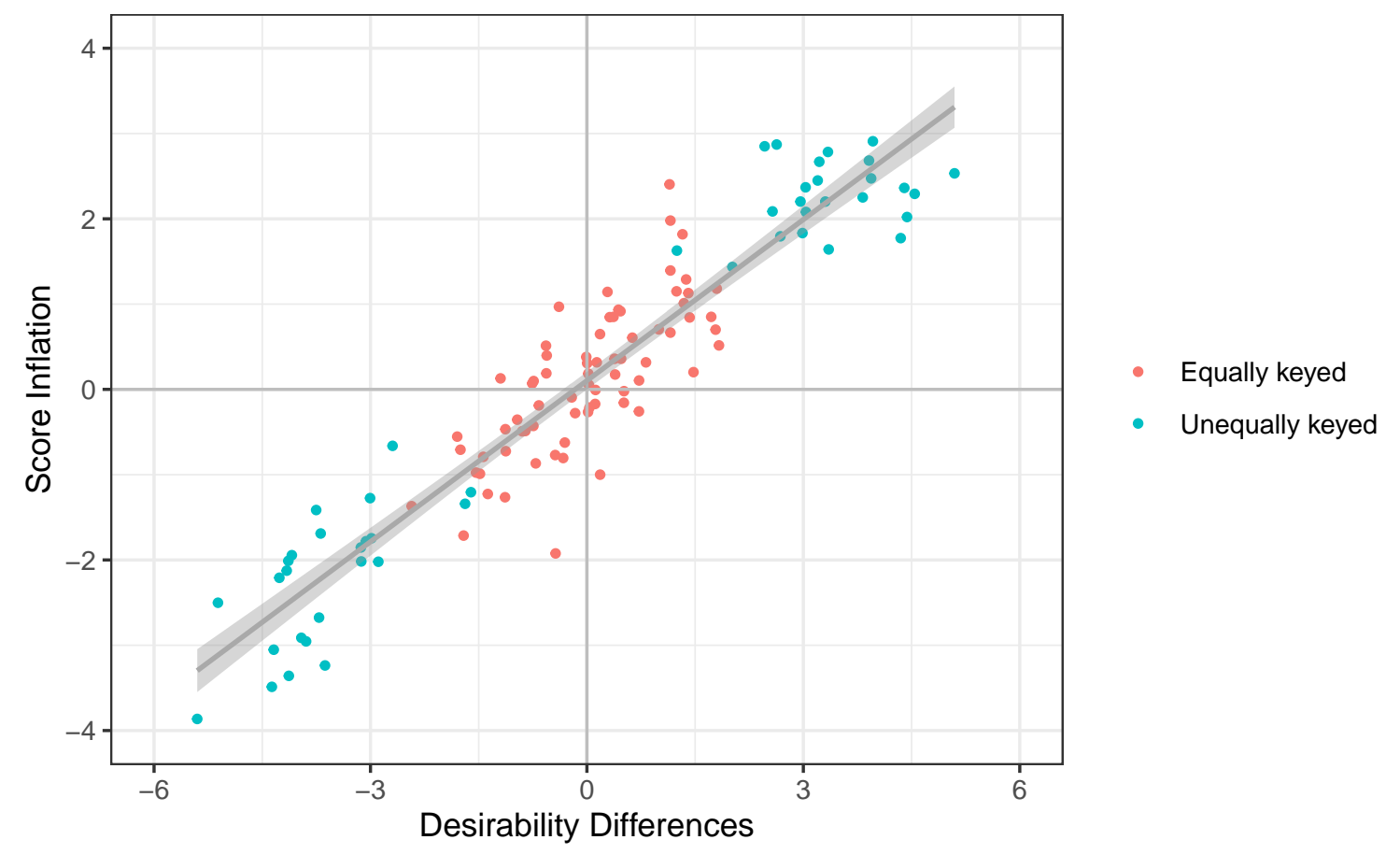

Figure 3

Scatter Plot and Unstandardized Regression Line for the Influence of Desirability Difference on Score Inflation.

\section{Explorative Analyses}

In the following, we report analyses that we performed in knowledge of the results presented above and that were not pre-registered. In this context, we are particularly interested in the counterintuitive results on the interaction of honest scores and individual faking tendency in the prediction of faking scores as well as the influence of item desirability on response behavior.

In Figure 2, GPC honest and faked scores are mostly negatively associated when faking tendency is high. We wanted to analyze these effects more closely. Further, we aimed to exclude the possibility that this is a statistical artifact; potential non-linear effects are represented exclusively by the interaction term, meaning that the data was potentially modeled inadequately. Therefore, we allowed for linear and quadratic effects in both predictors and their error variances. Figure 4 describes the same association with the faking tendency as a continuous variable and from a different perspective. The color describes the value of the faking score, and the lines run along constant values. Two different types of respondents reached high faking scores: first, those with a high faking tendency and low honest scores and, second, those with high honest scores but a low faking tendency. Those who showed medium levels of both predictors or even high levels of both predictors seem to yield lower faking scores than those with very high honest scores and a very low faking tendency or vice versa. Even though the patterns are very consistent across the traits, the 

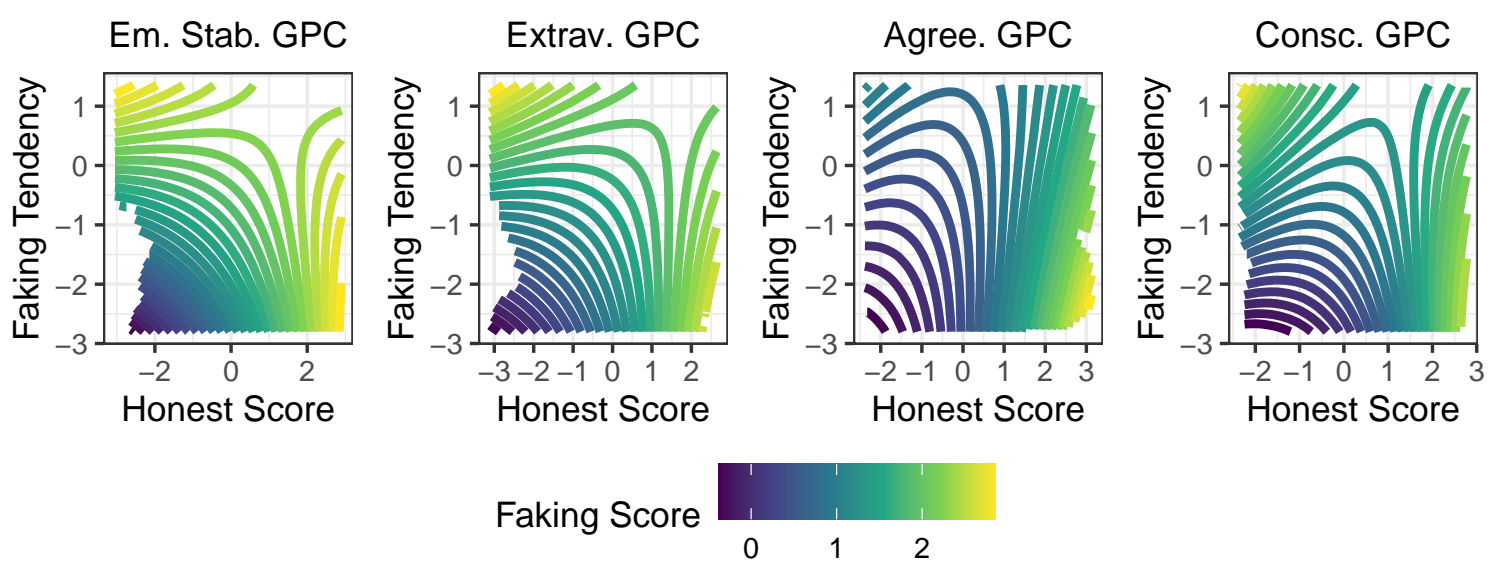

Figure 4

GPC Interaction Plots for Hypothesis 3 with Continuous Variables and Quadratic Effects. Em. Stab. = emotional stability; Extrav. = extraversion; Agree. = agreeableness; Consc $=$ conscientiousness; GPC = graded paired comparison condition. Lines end when information is not sufficient for parameter estimation.

results have to be interpreted with caution because the trait-wise credibility intervals of the honest scores' quadratic effects all include zero.

We also conducted additional analyses regarding social desirability. Results for Hypothesis 5 suggest that removing the unequally keyed items from the GPC questionnaire might reduce its fakeability. As can be seen in the lower four rows of Table 1, the GPCs achieve very good reliability even when only equally keyed item pairs are included. Based on this, we ran the regression models for Hypotheses 1 to 4 again with the GPC questionnaire, which this time was based exclusively on equally keyed items. The Likert questionnaire remained unchanged. The results of these analysis are presented in the online supplement. They do not indicate a better faking resistance of the GPCs when unequally keyed item pairs are removed. However, in the faking condition, responses to unequally keyed GPCs tended to be much more extreme than to equally keyed items; that is, the absolute difference of the GPC mean from the midpoint of the scale was much larger for unequally than for equally keyed items $(\Delta M=1.82,95 \%$ CI $[1.61,2.04], t(101.04)=16.72, p<.001$ for the difference of differences on a 9 -point scale).

As stated above, test developers often try to improve the faking resistance of FC questionnaires by matching items within a comparison (or, more generally, an item block) for social desirability. A widely used matching method is to use item means as readily available indicators of item desirability. However, it is questionable whether item means are a sufficient substitute for a direct measurement of item social desirability, since they are also influenced by other factors. For instance, a person with a fixed trait level can agree with two items of the same trait to different degrees, because complete agreement with these items requires different trait levels. An example would be the item "I have nothing against parties" vs. "I am the life of the party". Indeed, in our data, the item means of the honest 
condition were only correlated by $r=.37,95 \%$ CI $[.07, .60], t(40)=2.48, p=.017$ with the direct item desirability ratings. The fact that the item desirability ratings determine the actual response behavior is shown by their correlation of $r=.99,95 \%$ CI $[.99,>.99]$, $t(40)=49.64, p<.001$ with the item means of the Likert scale faking condition.

However, the isolated social desirability ratings of individual items must also be viewed with caution. In addition to the isolated ratings of social desirability of individual items, we had the respondents assess the relative social desirability of the two items from 28 paired comparisons. In fact, the differences of the isolated ratings correlate to $r=.89$, $95 \%$ CI $[.77, .95], t(26)=9.82, p<.001$ with the differences in social desirability of both items in a direct paired comparison. The correlation is high but not perfect, with the differences possibly being due to context effects of the other item in the pair comparison (and measurement error). The mean difference of desirability differences within one item pair measured with isolated desirability ratings vs. a direct paired comparison item desirabilities was 0.44 on a 7 -point scale ${ }^{2}$.

Another important implicit assumption for the composition of items based on their social desirability is that the composition is equally suitable for all respondents. Thus, the critical point is not whether items are perceived as equally attractive on average but whether each individual perceives them as equally attractive. This leads to the question of how much the perception of the social desirability of an item differs between individuals. The average standard deviation of an item's social desirability rating is $S D=1.15$ in our sample. Suppose we could always match two items with exactly the same average social desirability $\left(\mu_{1}-\mu_{2}=0\right)$. Further, for illustrative purposes we assume that the desirability ratings are multivariate normally distributed and uncorrelated. Then, the individual perception of differences in social desirability between two items is normally distributed with $M=$ $\mu_{1}-\mu_{2}=0$ and $S D=\sqrt{\sigma_{1}^{2}+\sigma_{2}^{2}}=\sqrt{1.15^{2}+1.15^{2}}=1.62$. Thus, even with items perfectly matched for the mean item social desirability, $32 \%$ of participants (those outside the limits of $[-1,1]$ of the normal distribution) would perceive differences in item desirability larger than 1.62 on a 7 -point scale. Such differences will likely lead to considerable faking behavior (see Figure 3).

\section{Discussion}

The main purpose of this study was to judge the utility of GPCs for personality assessment in high-stakes situations such as personnel selection. As expected, participants managed to fake both Likert and GPC scales, i.e., the mean trait scores were higher when respondents tried to distort them than when they answered honestly. However, the mean score inflation was lower in GPCs compared with Likert scales. Remarkably, the reduced score inflation did not translate into a closer association of honest and faked trait scores. Even though the scores are less inflated, they do not seem to capture the real differences between respondents more adequately in situations where they are motivated to distort. Thus, the assertion that GPCs would exhibit a higher faking resistance was only partially

\footnotetext{
${ }^{2}$ The desirability difference of isolated ratings is the difference between two desirability ratings on 7-point Likert scales. The desirability difference within a GPC is the difference from the scale midpoint of a 9-point paired comparison multiplied by $\frac{7}{9}$ to adjust for different scale lengths.
} 
supported. One factor on the respondent side that influences the association of honest and faked scores is the individual faking tendency. In both questionnaire types, scores can be manipulated if the respondent tries to do so. In Likert scales, the faking scores increased with higher faking tendencies and higher honest score. If one of the two (faking tendency or honest score) was highly pronounced, the other showed little additional effects. In contrast, in GPCs faking scores were the highest when either the honest scores or the faking tendency was high and the other variable was low. The association of honest and faked trait scores was even negative in individuals with low honest scores, especially when the faking tendency was high. Moreover, the association of these variables in GPC questionnaires could be non-linear, but our evidence is not conclusive in this respect. Finally, an extremely important questionnaire feature that determines the response inflation in a given GPC is the desirability difference between the two items involved. This desirability difference is much larger in unequally keyed item pairs than in equally keyed ones.

\section{Theoretical Implications}

The results confirm and expand our knowledge about the FC format in general and GPCs in particular. The meta-analytic finding that FC scores are inflated when respondents are motivated to fake but less so than in Likert questionnaires (Cao \& Drasgow, 2019) seems to apply to GPCs as well. The effect sizes we found were high and - if no further precautions are taken to avoid faking - faked scores are hardly associated with honest scores. Compared with most other faking studies and meta-analytic estimates, our effect sizes are relatively large. This might be caused by our instruction to participants, which was to present oneself as positively as possible. Faking effects are typically higher in instructionally induced designs than in studies with real-life motivational distortion, and having participants answer honestly before they distort their responses yields larger effect sizes than the opposite order (Edens \& Arthur, 2000). Even though our effect sizes reveal how much people are able to distort, they are likely often lower in practice. Thus, while our results are rather representative for people applying to very attractive jobs who are willing to show high levels of impression management in order to be selected they raise questions about the construct validity of personality tests in high-stakes situations. Specifically, meta-analyses (e.g., Schmidt \& Hunter, 1998; Barrick \& Mount, 1991) may overestimate the actual predictive validity of personality tests in high-stakes situations: A closer look at corresponding studies shows that they rely on participants already employed by a company, meaning they may have a lower faking motivation than actual job applicants.

The lower correlation between honest and faked trait scores for FC formats compared with Likert items has previously been observed for discrete FC formats (Guan, 2015; Heggestad et al., 2006; Pavlov et al., 2019). However, considering our study's design and analysis strategy, we can rule out alternative interpretations mentioned in earlier studies. Particularly, in our study neither the ipsative CTT scoring method (Heggestad et al., 2006) nor the lower reliability of discrete FC formats (Guan, 2015; Pavlov et al., 2019) are valid explanations. Instead, respondents with medium or high honest scores reached systematically lower scores in the applicant condition than those who had low true, i.e., honest, scores. The negative association of honest and faked scores in a subpopulation diminishes 
the overall correlation for the full population. Furthermore, non-linear effects might negatively influence the level of correlation. Together with the diminished correlations between honest and faked scores in FC questionnaire reported previously (Guan, 2015; Heggestad et al., 2006; Pavlov et al., 2019), these observations point to a severe psychometric weakness of the FC format. We assume that this effect is caused by artifacts of the interdependent nature of the response process and/or format. Each response change in favor of one item inevitably causes a change in the response to another item and, therefore, the corresponding trait score estimate. The consequences of these manipulations are difficult to foresee for respondents and might unintentionally negatively effect other traits' scores that they did not intend to affect.

Our results also give insights into the largely unexplored faking process in FC items. It seems as if the social desirability is crucial in this respect. Desirability differences between items and the score inflation in this item pair are very closely associated. Thus, if the difference between the desirability of two items in a given situation is known, it can be used to predict almost perfectly how much these items are distorted. Moreover, FC questionnaires have the potential to reduce mean score inflation to an absolute minimum if item desirabilities are exactly matched. This is in line with meta-analytic results, whereby FC questionnaires that underwent a matching procedure in the constructional process were superior to questionnaires that were not matched concerning social desirability (Cao \& Drasgow, 2019).

In addition, our results suggest very clearly that unequally keyed item pairs are faked particularly strongly. The responses to these item pairs changed considerably more between honest and faking conditions than did responses to equally keyed items. This is most likely due to the large differences in desirability of these item pairs. The present study provides empirical support for the assumption that in unequally keyed item pairs, one item typically represents the desired end and one the undesired end of a trait continuum, resulting in large desirability differences (Bürkner et al., 2019; Schulte et al., 2020).

Based on the high score inflation in unequally keyed items, it would have been reasonable to assume that the score inflation in GPC factor scores could be reduced by removing these item pairs. However, this was not the case in our explorative analyses. This might be due to the extreme responses to these items under faking conditions. Items that are strongly faked by almost all respondents contribute little to precise trait score estimation under faking conditions. In simulation studies on discrete FC formats where no faking was assumed, they appear to be crucial for an acceptable level of reliability (Brown \& Maydeu-Olivares, 2011; Bürkner et al., 2019; Schulte et al., 2020). In contrast, using a more informative graded response format, this study reached high reliabilities even when using only the equally keyed version of the GPC questionnaire. However, the factor loadings of our questionnaire were slightly higher than those in the simulation studies, such that a direct comparison of reliabilities is not possible.

Another noteworthy observation from the present study relates to the faking differences between traits, especially with regard to agreeableness. Traits that were perceived as more desirable seemed to have been faked more strongly. Even though other studies have found similar patterns (e.g., Birkeland et al., 2006; Heggestad et al., 2006) a larger trait 
number would be necessary to better generalize this observation.

\section{Practical Implications}

GPCs represent an important extension of FC-based response formats. In particular, they seem to allow for highly reliable trait estimates even with only equally keyed item pairs, which successfully eliminates a major weakness of discrete FC formats (Bürkner et al., 2019; Schulte et al., 2020). In this respect, they are preferable to other discrete FC formats. Regarding their fakeability, it seems that GPCs have similar strengths and weaknesses as have been reported for discrete FC formats. They can reduce the score inflation in job applicants or other respondents who are motivated to fake in high-stakes situations, but for practical applications, they can currently only be recommended to a limited extent. GPCs seem to be less capable than Likert scales of recovering applicants' true rank order. Thus, these scales should not be used to make important decisions until these problems have been resolved. Further research must show whether this is possible.

When test developers attempt to construct FC questionnaires, they should pay particular attention to the social desirability of the items, as the measurement can only be expected to be relatively faking resistant if the items to be compared are equally socially desirable. Accordingly, questionnaire development should start with an item pool that is large enough to exclude items that cannot be paired based on their desirability. Unequally keyed item pairs/blocks should be avoided completely in high-stakes situations, as they are strongly faked. For the rest of the items, the question arises as to which method is best suited for social desirability matching. One widely used method is the use of Likert item means from an honestly responding sample; however, in our data, these item means were only moderately associated with direct item desirability ratings. On the one hand, this indicates socially desirable response behavior on Likert scales even under honest conditions. But it also shows that these honest mean values are an inadequate method for measuring social desirability. This is in contrast to the item means of the Likert faking condition, which are almost perfectly determined by the item social desirability.

Nonetheless, at least two problems remain even with optimal matching based on the average social desirability of an item. First, our explorative results suggest that social desirability might change somewhat in the context of the other item within a paired comparison, as was found in very early work on FC (Waters, 1965). To exclude contextual effects, one would either have to have all possible item combinations rated or test and replace pairs in an iterative process.

Second, individuals differ so much in their assessment of the social desirability of an item that the individually perceived desirability differences will often influence response behavior, even if the item is perfectly matched for mean item desirability. This is an issue that, to our knowledge, has not yet been addressed but has potentially major consequences

for response distortion. Since we do not know the individual perceptions regarding social desirability before testing and because individual item compositions would cause major additional problems such as sequence and context effects, etc., the questionnaire composition cannot be individualized. The resulting differences in perceived social desirability are presumably a major problem for the faking resistance of $\mathrm{FC}$ questionnaires that cannot be 
eliminated. It would be possible to exclude items that show a particularly high variance in perceived social desirability, but this could quickly lead to large reductions in the item pool. Therefore, it can be assumed that this criterion would be applied very cautiously in practice.

\section{Limitations and Future Research}

In our study, we were able to show that high reliability can be achieved with GPCs. To precisely quantify the benefit of the graded scale in GPCs in comparison with discrete FC formats, analytic approaches or simulations would be appropriate. Such methods would allow one to control for other influencing variables such as factor loadings, which were comparatively high in our questionnaire and positively influenced the reliability. Furthermore, simulations could examine potential biases in the estimation of inter-trait correlations and the normativity (vs. ipsativity) of GPC factor scores as they have been found to be potentially biased or reduced in discrete FC formats (Schulte et al., 2020).

Since sufficient levels of reliability can be achieved with GPCs, the most severe remaining weakness of GPCs (and FC formats in general) seems to be their limited ability to recover the true rank order of responders' trait scores. Further research is necessary to determine why this is the case, and doing so would require gaining a better understanding of the faking processes in FC questionnaires. Although social desirability seems to play a major role here, the exact response processes and their consequences for the estimated factor scores remain largely unknown. Further, the negative effects of high faking intentions need to be examined in depth, as it would be interesting to learn whether this phenomenon is caused by the cognitive response process, the response format, response strategies specific to this format, or the analysis method.

Our study also indicates, but cannot conclusively ensure, that faking strength depends on the trait desirability. Further research on this question is needed because the potential consequences for test construction would be severe. Not only is it difficult to identify numerous traits that are all equally attractive in a given situation, but trait desirability also changes between situations. Thus, it would be impossible to construct high-dimensional questionnaires that are used for different application contexts (such as selection for different job profiles) by only analyzing selected dimensions.

Finally, as some of our results are explorative, they should be interpreted with caution until successfully replicated. This particularly concerns the findings on changed item desirabilities in the context of the other item in a pair. Even though this effect has been reported before (Waters, 1965), our results are based on few item pairs, and scale length varied between single item and paired measurement. Even though we do not assume this source of error variance to be large enough to explain the difference between individual

and combined ratings, future research should address these effects with an appropriate design in detail. 


\section{Conclusion}

To our knowledge, this study is the first to investigate the performance of the GPC format under high-stakes (i.e., faking) conditions. The graded response format can provide reliable personality measures and should, thus, be considered as an alternative to discrete FC formats. From a research perspective, it allows for more detailed insights into the general response distortion of FC response formats. Our study also indicates that some important aspects should be considered when designing faking-resistant FC questionnaires. The social desirability of the items - which in turn is extremely closely linked to item keying - is of outstanding importance. Social desirability has a very high impact on response behavior and score inflation. Furthermore, the faking effects we found resemble those of discrete FC formats, in that FC questionnaires constructed according to current knowledge can reduce score inflation but do not seem to outperform Likert scales in the recovery of applicants' true rank orders. Although the mean score inflation paradigm has previously dominated faking research, especially on FC questionnaires, it does not seem to account for important disadvantages of FC-based formats. If the effects found here are also found for discrete FC formats, which earlier studies suggest (Guan, 2015; Heggestad et al., 2006; Pavlov et al., 2019), then the relative fakeability of FC vs. Likert scales should be revised. To judge whether the issues we found can be resolved by improved questionnaire design and/or alternative analysis methods, a better understanding of the faking process and its effects on trait estimates is necessary.

\section{References}

Agresti, A. (1992). Analysis of ordinal paired comparison data. Journal of the Royal Statistical Society: Series C (Applied Statistics), 41(2), 287-297. https://doi.org/10. $2307 / 2347562$

Alfaro-Rodriguez, H., O’Mahony, M., \& Angulo, O. (2005). Paired preference tests: D' values from mexican consumers with various response options. Journal of Sensory Studies, 20(3), 275-281. https://doi.org/10.1111/j.1745-459X.2005.00018.x

Barrick, M. R., \& Mount, M. K. (1991). The big five personality dimensions and job performance: A meta-analysis. Personnel Psychology, 44(1), 1-26. https://doi.org/ 10.1111/j.1744-6570.1991.tb00688.x

Birkeland, S. A., Manson, T. M., Kisamore, J. L., Brannick, M. T., \& Smith, M. A. (2006). A meta-analytic investigation of job applicant faking on personality measures. International Journal of Selection and Assessment, 14(4), 317-335. https://doi.org/ 10.1111/j.1468-2389.2006.00354.x\%20

Brown, A., Inceoglu, I., \& Lin, Y. (2017). Preventing rater biases in 360-degree feedback by forcing choice. Organizational Research Methods, 20(1), 121-148. https://doi.org/ $10.1177 / 1094428116668036$

Brown, A., \& Maydeu-Olivares, A. (2011). Item response modeling of forced-choice questionnaires. Educational and Psychological Measurement, 71(3), 460-502. https: //doi.org/10.1177/0013164410375112 
Brown, A., \& Maydeu-Olivares, A. (2018). Ordinal factor analysis of graded-preference questionnaire data. Structural Equation Modeling, 25(4), 516-529. https://doi.org/10. 1080/10705511.2017.1392247

Bürkner, P.-C. (2017). brms: An R package for Bayesian multilevel models using Stan. Journal of Statistical Software, 80(1), 1-28. https://doi.org/10.18637/jss.v080.i01

Bürkner, P.-C. (2018). Advanced Bayesian multilevel modeling with the R package brms. The R Journal, 10(1), 395-411. https://doi.org/10.32614/RJ-2018-017

Bürkner, P.-C. (2020). ThurstonianIRT: Thurstonian irt models. https://CRAN.R-project. org $/$ package $=$ thurstonianIRT

Bürkner, P.-C., Schulte, N., \& Holling, H. (2019). On the statistical and practical limitations of thurstonian irt models. Educational and Psychological Measurement, 79(5), 827-854. https://doi.org/10.1177/0013164419832063

Cao, M., \& Drasgow, F. (2019). Does forcing reduce faking? A meta-analytic review of forced-choice personality measures in high-stakes situations. Journal of Applied Psychology, 104(11), 1347-1368. https://doi.org/10.1037/apl0000414

Carpenter, B., Gelman, A., Hoffman, M., Lee, D., Goodrich, B., Betancourt, M., Brubaker, M. A., Guo, J., Li, P., \& Ridell, A. (2017). Stan: A probabilistic programming language. Journal of Statistical Software, 76(1).

Cattell, R. B. (1944). Psychological measurement: Normative, ipsative, interactive. Psychological Review, 51(5), 292-303. https://doi.org/10.1037/h0057299

Chalmers, R. P. (2012). mirt: A multidimensional item response theory package for the R environment. Journal of Statistical Software, 48(6), 1-29. https://doi.org/10.18637/ jss.v048.i06

Christiansen, N. D., Burns, G. N., \& Montgomery, G. E. (2005). Reconsidering forcedchoice item formats for applicant personality assessment. Human Performance, 18(3), 267-307. https://doi.org/10.1207/s15327043hup1803_4

Clemans, W. V. (1966). An analytical and empirical examination of some properties of ipsative measures (Psychometrika Monograph No. 14). Psychometric Society. https: //www.psychometricsociety.org/sites/default/files/pdf/MN14.pdf

Dalal, D. K., Zhu, X., Rangel, B., Boyce, A. S., \& Lobene, E. (2019). Improving applicant reactions to forced-choice personality measurement: Interventions to reduce threats to test takers' self-concepts. Journal of Business and Psychology. https://doi.org/10. 1007/s10869-019-09655-6\%20

De Beuckelaer, A., Kampen, J. K., \& Van Trijp, J. C. M. (2013). An empirical assessment of the cross-national measurement validity of graded paired comparisons. Quality \&6 Quantity: International Journal of Methodology, 47(2), 1063-1076. https://doi.org/ $10.1007 / \mathrm{s} 11135-011-9583-1$

Donovan, J. J., Dwight, S. A., \& Hurtz, G. M. (2003). An assessment of the prevalence, severity, and verifiability of entry-level applicant faking using the randomized 
response technique. Human Performance, 16(1), 81-106. https://doi.org/10.1207/ S15327043HUP1601_4

Edens, P., \& Arthur, W. (2000). A meta-analysis investigating the susceptibility of selfreport inventories to distortion. 15th Annual Conference of the Society for Industrial and Organizational Psychology, New Orleans, La.

Griffin, B., \& Wilson, I. G. (2012). Faking good: Self-enhancement in medical school applicants. Medical Education, 46(5), 485-490. https://doi.org/10.1111/j.1365-2923. 2011.04208.x

Griffith, R. L., Chmielowski, T., \& Yoshita, Y. (2007). Do applicants fake? An examination of the frequency of applicant faking behavior. Personnel Review, 36 (3), 341-355. https: //doi.org/10.1108/00483480710731310

Guan, L. (2015). Personality, faking, and the ability of identify criteria: Can forced choice formats untangle their relationships? [Unpublished master thesis]. University of Virginia.

Guenole, N., Brown, A., \& Cooper, A. J. (2018). Forced-choice assessment of work-related maladaptive personality traits: Preliminary evidence from an application of thurstonian item response modeling. Assessment, 25(4), 513-526. https://doi.org/10.1177/ 1073191116641181

Heggestad, E. D., Morrison, M., Reeve, C. L., \& McCloy, R. A. (2006). Forced-choice assessments of personality for selection: Evaluating issues of normative assessment and faking resistance. Journal of Applied Psychology, 91(1), 9-24. https://doi.org/10. 1037/0021-9010.91.1.9

Hicks, L. E. (1970). Some properties of ipsative, normative, and forced-choice normative measures. Psychological Bulletin, 74(3), 167-184. https://doi.org/10.1037/h0029780

Hontangas, P. M., Torre, J. de la, Ponsoda, V., Leenen, I., Morillo, D., \& Abad, F. J. (2015). Comparing traditional and irt scoring of forced-choice tests. Applied Psychological Measurement, 39(8), 598-612. https://doi.org/10.1177/0146621615585851

Huber, C. R. (2017). Faking and the validity of personality tests: Using new fakingresistant measures to study some old questions [Doctoral dissertation, University of Minnesota]. https://conservancy.umn.edu/bitstream/handle/11299/185605/Huber umn_0130E_17909.pdf? sequence=1\&isAllowed=y

Jackson, D. N., Wroblewski, V. R., \& Ashton, M. C. (2000). The impact of faking on employment tests: Does forced choice offer a solution?. Human Performance, 13(4), 371-388. https://doi.org/10.1207/S15327043HUP1304_3

Johnson, C. E., Wood, R., \& Blinkhorn, S. F. (1988). Spuriouser and spuriouser: The use of ipsative personality tests. Journal of Occupational Psychology, 61 (2), 153-162. https://doi.org/10.1111/j.2044-8325.1988.tb00279.x

Lee, J. W., Jones, P. S., Mineyama, Y., \& Zhang, X. E. (2002). Cultural differences in responses to a likert scale. Research in Nursing \& Health, 25(4), 295-306. https: //doi.org/10.1002/nur.10041 
Lee, P., Joo, S.-H., \& Lee, S. (2019). Examining stability of personality profile solutions between likert-type and multidimensional forced choice measure. Personality and Individual Differences, 142, 13-20. https://doi.org/10.1016/j.paid.2019.01.022

Likert, R. (1932). A technique for the measurement of attitudes. Archives of Psychology, 22, 5-55. https://legacy.voteview.com/pdf/Likert_1932.pdf

McFarland, L. A., \& Ryan, A. M. (2000). Variance in faking across noncognitive measures. Journal of Applied Psychology, 85(5), 812-821. https://doi.org/10.1037/0021-9010.85. 5.812

Ofir, C. (2004). Reexamining latitude of price acceptability and price thresholds: Predicting basic consumer reaction to price. Journal of Consumer Research, 30(4), 612-621. $10.1086 / 380293$

Paulhus, D. L., \& Jones, D. N. (2014). Measurement of dark personalities. In G. J. Boyle, D. H. Saklosfske, \& G. Matthews (Eds.), Measures of personality and social psychological constructs (pp. 562-594). Academic Press. https://doi.org/10.1016/ B978-0-12-386915-9.00020-6

Paulhus, D. L., \& Vazire, S. (2007). The self-report method. In R. W. Robins, R. C. Fraley, \& R. F. Krueger (Eds.), Handbook of research methods in personality psychology (pp. 224-239). Guilford.

Pavlov, G. (2015). Intentional response distortion effects on personality scores in simulated personnel assessment settings: A moderation study [Doctoral dissertation, ie University, Madrid, Spain]. https://www.researchgate.net/publication/307599102_ Intentional_Response_Distortion_Effects_on_Personality_Scores_in_Simulated_ Personnel_Assessment_Settings_A_Moderation_Study

Pavlov, G., Maydeu-Olivares, A., \& Fairchild, A. J. (2019). Effects of applicant faking on forced-choice and likert scores. Organizational Research Methods, 22(3), 710-739. https://doi.org/10.1177/1094428117753683

R Core Team. (2020). R: A language and environment for statistical computing. R Foundation for Statistical Computing. https://www.R-project.org/

Rosse, J. G., Stecher, M. D., Miller, J. L., \& Levin, R. A. (1998). The impact of response distortion on preemployment personality testing and hiring decisions. Journal of Applied Psychology, 83(4), 634-644. https://doi.org/10.1037/0021-9010.83.4.634

Samejima, F. (1969). Estimation of latent ability using a response pattern of graded scores. Psychometrika Monograph Supplement, 34(4, Pt. 2), 100. http://search.ebscohost.com/login.aspx?direct=true\&db=psyh\&AN=197204809-001\&lang=de\&site=ehost-live

Saville, P., \& Willson, E. (1991). The reliability and validity of normative and ipsative approaches in the measurement of personality. Journal of Occupational Psychology, 64(3), 219-238. https://doi.org/10.1111/j.2044-8325.1991.tb00556.x

Schmidt, F. L., \& Hunter, J. E. (1998). The validity and utility of selection methods in personnel psychology: Practical and theoretical implications of 85 years of research 
findings. Psychological Bulletin, 124(2), 262-274. https://doi.org/10.1037/0033-2909. 124.2.262

Scholz, S. W., Meissner, M., \& Decker, R. (2010). Measuring consumer preferences for complex products: A compositional approach based on paired comparisons. Journal of Marketing Research, 47(4), 685-698. https://doi.org/10.1509/jmkr.47.4.685

Schulte, N., Holling, H., \& Bürkner, P.-C. (2020). Can high-dimensional questionnaires resolve the ipsativity issue of forced-choice response formats? Educational and Psychological Measurement. https://doi.org/10.1177/0013164420934861

Skedgel, C. D., Wailoo, A. J., \& Akehurst, R. L. (2015). Choosing vs allocating: Discrete choice experiments and constant-sum paired comparisons for the elicitation of societal preferences. Health Expectations: An International Journal of Public Participation in Health Care $\&$ Health Policy, 18(5), 1227-1240. https://doi.org/10.1111/hex.12098

Vasilopoulos, N. L., Cucina, J. M., Dyomina, N. V., Morewitz, C. L., \& Reilly, R. R. (2006). Forced-choice personality tests: A measure of personality and cognitive ability? Human Performance, 19 (3), 175-199. https://doi.org/10.1207/s15327043hup1903_1

Viswesvaran, C., \& Ones, D. S. (1999). Meta-analyses of fakability estimates: Implications for personality measurement. Educational and Psychological Measurement, 59(2), 197210. https://doi.org/10.1177/00131649921969802

Wang, W.-C., Qiu, X.-L., Chen, C.-W., Ro, S., \& Jin, K.-Y. (2017). Item response theory models for ipsative tests with multidimensional pairwise comparison items. Applied Psychological Measurement, 41(8), 600-613. https://doi.org/10.1177/ 0146621617703183

Waters, L. K. (1965). A note on the "fakability" of forced-choice scales. Personnel Psychology, 18(2), 187-191. https://doi.org/10.1111/j.1744-6570.1965.tb00277.x\%20

Wetzel, E., Böhnke, J. R., \& Brown, A. (2016). Response biases. In F. T. L. Leong, D. Bartram, F. M. Cheung, K. F. Geisinger, \& D. Iliescu (Eds.), The itc international handbook of testing and assessment (pp. 349-363). Oxford University Press Oxford.

Wetzel, E., \& Greiff, S. (2018). The world beyond rating scales: Why we should think more carefully about the response format in questionnaires. European Journal of Psychological Assessment, 34 (1), 1-5. https://doi.org/10.1027/1015-5759/a000469

Young, A. L. (2018). Faking resistance of a forced-choice measure of the dark triad [Doctoral dissertation, North Carolina State University]. https://repository.lib.ncsu.edu/ bitstream/handle/1840.20/35649/etd.pdf?sequence=1

Zhang, B., Cao, M., Tay, L., Luo, J., \& Drasgow, F. (2020). Examining the item response process to personality measures in high-stakes situations: Issues of measurement validity and predictive validity. Personnel Psychology, 73(2), 305-332. https://doi.org/10.1111/peps.12353 